\title{
APPENDIX I. GRAIN SIZE ANALYSES, LEG 17
}

\author{
Gerald W. Bode, Scripps Institution of Oceanography, La Jolla, California
}

Sand-silt-clay distribution was determined on 10-cc sediment samples collected at the time the cores were split and described. The results are listed in Table 1.

The sediment classification used here is that of Shepard (1954) with the sand, silt, and clay boundaries based on the Wentworth (1922) scale (Figure 1). Thus the sand, silt, and clay fractions are composed of particles whose diameters range from 2000 to 62.5 microns, 62.5 to 3.91 microns, and less than 3.91 microns, respectively. This classification is applied regardless of sediment type and origin; therefore, the sediment names used in this table may differ from those used elsewhere in this volume, e.g., a silt composed of nannofossils in this table may be called a nanno ooze in a site chapter.

Standard sieve and pipette methods were used to determine the grain size distribution. The sediment sample was dried and dispersed in a Calgon solution, and if a sediment sample failed to disaggregate, it was treated with a sonic probe and, if necessary, hyrdogen peroxide. Sediment samples which resisted the above treatment were not analyzed.

The sand-size fraction was removed by wet sieving using a 63-micron sieve, and the silt and clay fractions were analyzed by standard pipette analysis. Sampling depths and times were calculated using equations derived from Stokes settling velocity equation (Krumbein and Pettijohn, 1938, p. 95-96):

$$
\begin{aligned}
& \frac{D}{t}=V \frac{2\left(d_{1}-d_{2}\right) \mathrm{g} r^{2}}{9 n} \\
& t=\frac{9 D \eta}{2 \mathrm{~g} r^{2}\left(d_{1}-d_{2}\right)}
\end{aligned}
$$

where

$$
\begin{aligned}
V= & \text { velocity, in } \mathrm{cm} / \mathrm{sec} \\
t^{*}= & \text { time, in sec } \\
D= & \text { depth pipette is inserted, in } \mathrm{cm} \\
\mathrm{g}^{*}= & \text { gravity, in } \mathrm{cm} / \mathrm{sec}^{2} \\
r^{*}= & \text { radius of individual particles, in cm } \\
d_{1}= & \text { density of solid particles arbitrarily set at } 2.675 \\
& \mathrm{~g} / \mathrm{cc} \\
d_{2}= & \text { absolute density of distilled water at different } \\
& \text { temperatures (Hodgman et al., 1960, p. 2129) }
\end{aligned}
$$

$$
\begin{aligned}
\eta^{*}= & \text { viscosity of distilled water in poises at different } \\
& \text { temperatures (Hodgman et al., 1960, p. 2181). }
\end{aligned}
$$

\footnotetext{
*Five figures were used in calculations to avoid rounding off variations.
}

The reproducibility of the grain size analyses has been previously tested (Boyce, 1972), and it was found that over a period of time with several operators the reproducibility for the sand-silt-clay fractions is $\pm 2.5 \%$ (absolute). For detailed step-by-step procedures, see Volume IV of the Initial Reports of the Deep Sea Drilling Project.

\section{REFERENCES}

Boyce, R. E., 1972. Grain Size Analysis, Leg 9, Deep Sea Drilling Project: Initial Reports of the Deep Sea Drilling Project, Volume IX. Washington (U.S. Government Printing Office), p. 779.

Hodgman, C. D., Weast, R. C., and Selby, S. M., 1960, Handbook of Chemistry and Physics: Cleveland (Chemical Rubber Publishing Co.), 3472 p.

Krumbein, W. C. and Pettijohn, F. J., 1938, Manual of Sedimentary Petrography: New York (Appleton Century Cor.), 549 p.

Shepard, F. P., 1954. Nomenclature based on sand-silt-clay ratios: J. Sediment. Petrol., v. 24, p. 151.

Wentworth, C. K., 1922. A scale of grade and class terms for clastic sediments: J. Geol., v. 30, p. 377.

TABLE 1

Grain Size Determinations, Leg 17

\begin{tabular}{llllll}
\hline $\begin{array}{c}\text { Core Section, } \\
\text { Top of }\end{array}$ & & & & & \\
Interval & Depth & Sand & Silt & Clay & \\
$(\mathrm{cm})$ & $(\mathrm{cm})$ & $(\%)$ & $(\%)$ & $(\%)$ & Classification \\
\hline
\end{tabular}

Site 164

$\begin{array}{lrrrrl}10-4,21.0 & 116.7 & 0.7 & 28.3 & 71.0 & \text { Silty clay } \\ 12-1,99.0 & 132.0 & 0.8 & 23.6 & 75.6 & \text { Clay } \\ 13-1,133.0 & 138.3 & 0.2 & 23.8 & 76.0 & \text { Clay } \\ 17-1,86.0 & 178.9 & 57.1 & 20.2 & 22.7 & \text { Sand-silt-clay } \\ 25-1,122.0 & 253.2 & 41.5 & 23.5 & 34.9 & \text { Sand-silt-clay }\end{array}$

Site 165

$\begin{array}{llllll}2-1,129.0 & 6.3 & 2.8 & 63.2 & 34.0 & \text { Clayey silt }\end{array}$

Site 165A

\begin{tabular}{rrrrrl}
$2-1,54.0$ & 14.5 & 1.6 & 61.8 & 36.6 & Clayey silt \\
$2-3,14.0$ & 17.1 & 2.2 & 52.9 & 44.9 & Clayey silt \\
$2-3,60.0$ & 17.6 & 7.1 & 60.4 & 32.5 & Clayey silt \\
$2-3,80.0$ & 17.8 & 7.5 & 67.9 & 24.6 & Clayey silt \\
$2-3,96.0$ & 18.0 & 18.0 & 57.9 & 24.1 & Clayey silt \\
\hline
\end{tabular}


TABLE 1 - Continued

\begin{tabular}{lrrrrl}
\hline $\begin{array}{c}\text { Core Section, } \\
\text { Top of } \\
\text { Interval } \\
(\mathrm{cm})\end{array}$ & $\begin{array}{c}\text { Depth } \\
(\mathrm{cm})\end{array}$ & $\begin{array}{c}\text { Sand } \\
(\%)\end{array}$ & $\begin{array}{r}\text { Silt } \\
(\%)\end{array}$ & $\begin{array}{c}\text { Clay } \\
(\%)\end{array}$ & Classification \\
\hline $8-1,15.0$ & 145.1 & 5.5 & 52.1 & 42.4 & Clayey silt \\
$8-1,34.0$ & 145.3 & 1.9 & 59.3 & 38.7 & Silt \\
$8-1,82.0$ & 145.8 & 11.3 & 84.6 & 4.1 & Silt \\
$8-1,102.0$ & 146.0 & 17.7 & 53.3 & 29.0 & Clayey silt \\
$8-6,72.0$ & 153.2 & 25.3 & 47.1 & 27.5 & Sand-silt-clay \\
$8-6,88.0$ & 153.4 & 10.2 & 57.0 & 32.8 & Clayey silt \\
$9-3,113.0$ & 158.1 & 15.4 & 42.6 & 42.0 & Clayey silt \\
$10-1,60.0$ & 201.6 & 2.3 & 46.5 & 51.1 & Silty clay \\
$10-4,115.0$ & 206.6 & 11.4 & 49.2 & 39.4 & Clayey silt \\
$11-3,128.0$ & 214.3 & 2.6 & 41.8 & 55.5 & Silty clay \\
$12-3,110.0$ & 223.1 & 3.4 & 43.7 & 53.0 & Silty clay \\
$12-5,20.0$ & 225.2 & 3.4 & 47.1 & 49.5 & Silty clay \\
$12-5,45.0$ & 225.4 & 11.1 & 57.4 & 31.5 & Clayey silt \\
$12-5,107.0$ & 226.1 & 0.7 & 67.0 & 32.4 & Clayey silt \\
$22-3,127.0$ & 400.3 & 3.3 & 45.5 & 51.2 & Silty clay \\
$23-2,34.0$ & 425.8 & 14.3 & 49.9 & 35.8 & Clayey silt
\end{tabular}

\section{Site 166}

$\begin{array}{llllll}14-2,70.0 & 180.2 & 10.4 & 40.4 & 49.1 & \text { Silty clay } \\ 25-1,127.0 & 271.3 & 11.7 & 47.9 & 40.4 & \text { Clayey silt }\end{array}$

Site 167

\begin{tabular}{lrrrrl}
$1-3,105.0$ & 4.0 & 24.2 & 32.5 & 43.4 & Sand-silt-clay \\
$1-5,136.0$ & 7.4 & 24.3 & 31.0 & 44.7 & Sand-silt-clay \\
$2-2,108.0$ & 11.6 & 27.4 & 29.5 & 43.1 & Sand-silt-clay \\
$2-6,80.0$ & 17.3 & 24.7 & 30.8 & 44.5 & Sand-silt-clay \\
$3-4,70.0$ & 23.2 & 11.4 & 26.0 & 62.5 & Silty clay \\
$3-5,50.0$ & 24.5 & 18.3 & 30.6 & 51.1 & Silty clay \\
$4-1,54.0$ & 66.5 & 4.7 & 35.4 & 59.8 & Silty clay \\
$4-3,72.0$ & 69.7 & 4.6 & 37.5 & 57.9 & Silty clay \\
$4-4,66.0$ & 71.2 & 8.6 & 39.6 & 51.7 & Silty clay \\
$4-6,100.0$ & 74.5 & 6.3 & 42.8 & 50.8 & Silty clay \\
$5-3,64.0$ & 106.6 & 3.2 & 47.2 & 49.6 & Silty clay \\
$6-4,80.0$ & 145.3 & 11.0 & 48.5 & 40.5 & Clayey silt \\
$7-2,90.0$ & 151.4 & 0.4 & 51.6 & 47.9 & Clayey silt \\
$7-5,103.0$ & 156.0 & 16.7 & 50.5 & 32.9 & Clayey silt \\
$7-5,147.0$ & 156.5 & 5.6 & 54.8 & 39.6 & Clayey silt \\
$8-1,124.0$ & 187.2 & 12.4 & 57.4 & 30.2 & Clayey silt \\
$9-1,110.0$ & 224.1 & 2.4 & 53.2 & 44.3 & Clayey silt \\
$9-2,52.0$ & 225.0 & 6.3 & 55.3 & 38.4 & Clayey silt \\
$9-4,88.0$ & 228.4 & 16.3 & 41.9 & 41.8 & Clayey silt \\
$10-6,88.0$ & 268.4 & 3.0 & 45.7 & 51.3 & Silty clay \\
$11-1,72.0$ & 297.7 & 3.8 & 59.3 & 37.0 & Clayey silt \\
$11-5,70.0$ & 303.7 & 4.2 & 49.0 & 46.8 & Clayey silt \\
$12-2,80.0$ & 336.3 & 5.7 & 48.4 & 45.9 & Clayey silt \\
$12-4,60.0$ & 339.1 & 3.1 & 53.5 & 43.5 & Clayey silt \\
$13-5,80.0$ & 376.8 & 11.9 & 64.5 & 23.6 & Clayey silt \\
$14-2,106.0$ & 409.6 & 6.7 & 52.2 & 41.1 & Clayey silt \\
$14-4,41.0$ & 411.9 & 7.6 & 41.7 & 50.7 & Silty clay \\
$15-5,127.0$ & 442.3 & 8.2 & 48.1 & 43.8 & Clayey silt \\
$18-2,80.0$ & 474.3 & 3.3 & 53.9 & 42.9 & Clayey silt \\
$19-2,80.0$ & 483.3 & 3.6 & 44.1 & 52.3 & Silty clay \\
\hline & & & & & \\
\hline
\end{tabular}

TABLE 1 - Continued

\begin{tabular}{lccrll}
\hline $\begin{array}{c}\text { Core, Section } \\
\text { Top of } \\
\text { Interval } \\
(\mathrm{cm})\end{array}$ & $\begin{array}{c}\text { Depth } \\
(\mathrm{cm})\end{array}$ & $\begin{array}{c}\text { Sand } \\
(\%)\end{array}$ & $\begin{array}{r}\text { Silt } \\
(\%)\end{array}$ & $\begin{array}{c}\text { Clay } \\
(\%)\end{array}$ & Classification \\
\hline $20-2,51.0$ & 493.0 & 2.8 & 47.6 & 49.6 & Silty clay \\
$21-5,60.0$ & 506.6 & 5.8 & 49.0 & 45.2 & Clayey silt \\
$22-1,80.0$ & 509.8 & 7.3 & 57.4 & 35.2 & Clayey silt \\
$23-2,30.0$ & 520.8 & 9.6 & 62.8 & 27.6 & Clayey silt \\
$23-5,106.0$ & 526.1 & 8.2 & 61.8 & 30.0 & Clayey silt \\
$28-2,40.0$ & 556.9 & 2.6 & 44.4 & 52.9 & Silty clay \\
$32-3,132.0$ & 596.3 & 3.5 & 45.4 & 51.1 & Silty clay \\
$39-1,125.0$ & 658.3 & 17.8 & 34.9 & 47.2 & Silty clay \\
$42-5,65.0$ & 691.7 & 10.7 & 43.6 & 45.6 & Silty clay \\
$45-6,93.0$ & 721.4 & 3.4 & 22.7 & 73.9 & Silty clay \\
$55-2,95.0$ & 807.5 & 1.1 & 33.8 & 65.1 & Silty clay \\
$57-1,148.0$ & 824.5 & 0.3 & 22.0 & 77.7 & Clay \\
$58-2,66.0$ & 829.2 & 0.1 & 9.7 & 90.2 & Clay \\
$58-3,15.0$ & 830.2 & 0.1 & 22.7 & 77.2 & Clay
\end{tabular}

Site 170

\begin{tabular}{llllll}
$2-2,51.0$ & 3.0 & 0.1 & 36.2 & 63.6 & Silty clay \\
\hline
\end{tabular}

Figure 1. Sediment classification after Shepard (1954) with the sand, silt, and clay size fractions based on the Wentworth (1922) grade scale: sand, silt, and clay size particles have respective diameters of 2000 to 62.5 microns, 62.5 to 3.91 microns, and <3.91 microns. Shepard's (1954) sediment classification is a function of sand, silt, and clay size particles and not composition. 